\title{
Descrição da técnica cirúrgica minimamente invasiva vídeo totalmente endoscópica interlaminar para tratamento de hérnia de disco lombar
}

\author{
Marco Aurélio Moscatelli Alvarenga1, Antônio Augusto Roth Vargas², \\ Marcelo Senna Xavier de Lima ${ }^{3}$, Paulo Roland Kaleff ${ }^{3}$
}

Fundação Centro Médico de Campinas, Campinas, SP. Santa Casa de Misericórdia de Limeira, Limeira, SP, Brasil.

\section{RESUMO}

Os autores descrevem a técnica minimamente invasiva de abordagem cirúrgica vídeo totalmente endoscópica (full-videoendoscopic) via posterior da coluna lombar para o tratamento de hérnia de disco lombar, podendo ser utilizada também para estenose de canal vertebral, estenose foraminal, cisto facetário, entre outras patologias. O sistema vídeo totalmente endoscópico transformou a cirurgia da coluna, apresentando um método muito menos traumático e com o mínimo de morbidade, comparado com as técnicas convencionais e videoendoscópicas assistidas, com resultados clínicos semelhantes. O procedimento pode ser realizado sob anestesia local associada a sedação ou anestesia geral, em sistema de hospital-dia. O procedimento é realizado totalmente sob a visão endoscópica, submerso em irrigação contínua de soro fisiológico, com visualização total das estruturas neurais, fragmentos de disco, facetas e forames. Com a utilização da videoendoscopia, estamos capacitados a realizar modernos procedimentos de forma eficaz, segura e precisa, com o menor índice de comorbidades, traumatismos e infecção.

\section{PALAVRAS-CHAVE}

Endoscopia, coluna vertebral, procedimentos cirúrgicos minimamente invasivos, descolamento do disco intervertebral.

\section{ABSTRACT}

Description of minimally invasive surgical techniques full-videoendoscopic interlaminar for treatment of herniation lumbar disc

The authors describe the technique of minimally invasive surgical full-videoendoscopic the posterior lumbar spine for the treatment of lumbar disc herniation can also be used for spinal canal stenosis, foraminal stenosis, facet cyst, among other diseases. The totally endoscopic video system made spine surgery, with a much less traumatic and with minimal morbidity compared with conventional techniques and video endoscopic assisted with similar outcomes. The procedure can be performed under local anesthesia with sedation or general anesthesia in the hospital system-day. The procedure is performed under endoscopic totally submerged in saline irrigation continues, with full visualization of the neural structures, disk fragments, facets and foramina. With the use of video endoscopic, we are able to perform modern procedures effectively, safely and accurately, with the lowest rate of comorbidities, trauma and infection.

\section{KEYWORDS}

Endoscopy, spine, surgical procedures minimally invasive, intervertebral disc displacement.

1 Médico-residente de Neurocirurgia da Fundação Centro Médico de Campinas, Campinas, SP, Brasil.

2 Professor titular da Residência em Neurocirurgia da Fundação Centro Médico de Campinas, Campinas, SP, Brasil e Santa Casa de Misericórdia de Limeira, Limeira, SP, Brasil.

3 Professor-associado da Residência em Neurocirurgia da Fundação Centro Médico de Campinas e Santa Casa de Misericórdia de Limeira, Limeira, SP, Brasil. 


\section{Introdução}

A cirurgia vídeo totalmente endoscópica (fullvideoendoscopic), um método já adotado há anos em outras especialidades e procedimentos, transformou a cirurgia da coluna, apresentando-se como um método muito menos traumático e com o mínimo de morbidade comparado com as técnicas convencionais e videoendoscópicas assistidas, com resultados clínicos semelhantes. O procedimento é realizado com a orientação fluoroscópica e visão vídeo totalmente endoscópica, por meio de um acesso minimamente invasivo. ${ }^{1} \mathrm{O}$ acesso à coluna vertebral lombar pode ser realizado por duas vias: a transforaminal, inicialmente criada para níveis lombares mais altos, por possuir dificuldades com o osso ilíaco; e a via de acesso interlaminar, criada posteriormente, para suprir também os acessos mais baixos como L4-L5 e L5-S1. ${ }^{1}$

\section{Indicações}

O procedimento está indicado para todos os quadros de hérnias discais lombossacras medianas, centro-laterais e foraminais, assim como para o tratamento de canal estreito lombar de um nível, foraminectomias, exérese de cistos facetários, discectomia percutânea com visualização direta do disco intervertebral, biópsias vertebrais. ${ }^{1}$

O fato de o procedimento poder ser realizado com anestesia local, sedação, ou geral possibilita a realização cirúrgica em pacientes com riscos cirúrgicos e anestésicos elevados, o qual em procedimentos com anestesia geral seria impossibilitado.

É indicado para pacientes com obesidade mórbida, pois, com o acesso videoendoscópico total, não importa o tamanho do panículo adiposo do paciente, diferente de um procedimento aberto.

\section{Contraindicações}

Além de contraindicações cirúrgicas gerais, o procedimento é contraindicado por falta ou deficiência na técnica cirúrgica referente ao médico cirurgião, em quadros de estenose de canal medular em mais de um nível, caso exista a necessidade de estabilização cirúrgica conjunta com discectomia, artrodeses multissegmentares e urgências compressivas como síndrome da calda equina e do cone medular. ${ }^{2}$

\section{Vantagens}

- Procedimento minimamente invasivo, com danos teciduais mínimos;

- Acesso mais rápido ao objetivo cirúrgico;

- Redução de sangramento com o fluxo contínuo de soro fisiológico através da óptica;

- Maior segurança cirúrgica com a visualização mais próxima das estruturas neurais;

- Tempo cirúrgico reduzido;

- Índice de infecção baixo ou nulo.

\section{Desvantagens}

- Limitação da mobilidade no interior do canal medular;

- Impossibilidade de rafia dural em caso de durotomia extensa;

- Material com custo mais elevado que o convencional;

- Dificuldade para reoperações por fibrose e perdas de parâmetros anatômicos;

- Visão em 2D.

\section{Procedimento cirúrgico}

O paciente é posicionado em decúbito ventral sobre coxins ou em mesa radiotransparente específica para cirurgias de coluna (Figura 1).

Realizar marcação radioscópica em dois planos, identificando nível objetivado. ${ }^{2}$

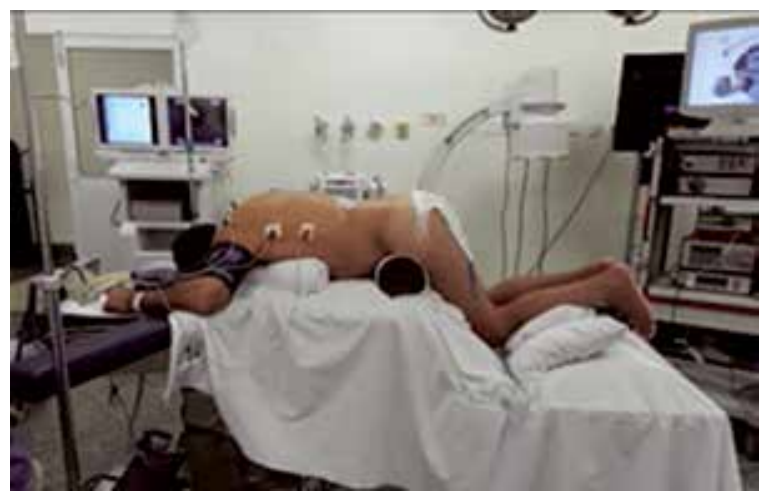

Figura 1 - Posicionamento cirúrgico. 


\section{Abordagem interlaminar}

Segundo Sebastian Ruetten, é realizada uma incisão paramediana próxima à linha média na pele em local previamente demarcado por fluoroscopia.

É inserido um dilatador de $6,9 \mathrm{~mm}$ de diâmetro em direção à janela interlaminar supraligamentar, com posterior verificação fluoroscópica (Figura 2).

Depois de inserido o dilatador, é colocado o canal de trabalho biselado com diâmetro de 7,9 mm direcionado para o ligamento amarelo (Figura 3).

Realiza-se o posicionamento da óptica através do canal de trabalho com irrigação contínua de soro fisiológico $0,9 \%$ (Figura 4).

Identificam-se das estruturas adjacentes (lâmina vertebral, ligamento amarelo, faceta articular), por meio do movimento de joystick com a mão não dominante (Figuras 4, 5 e 8).

Procede-se à abertura do ligamento amarelo com o auxílio de um saca-bocado, utilizando o canal de trabalho biselado para afastar as estruturas neurais adjacentes sob a visão endoscópica direta (Figuras 6 e 7).

Visualizado o abaulamento discal ou fragmento discal herniado, procede-se à abertura do ligamento, se necessário, e à retirada do fragmento discal (Figura 9).

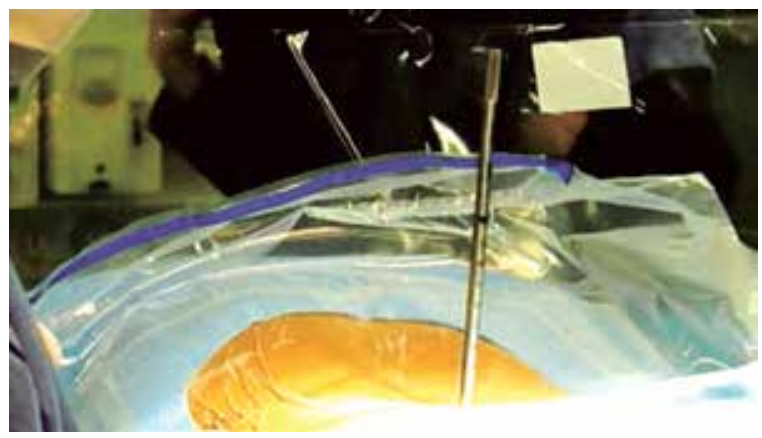

Figura 2 - Inserção de dilatador no nível demarcado.

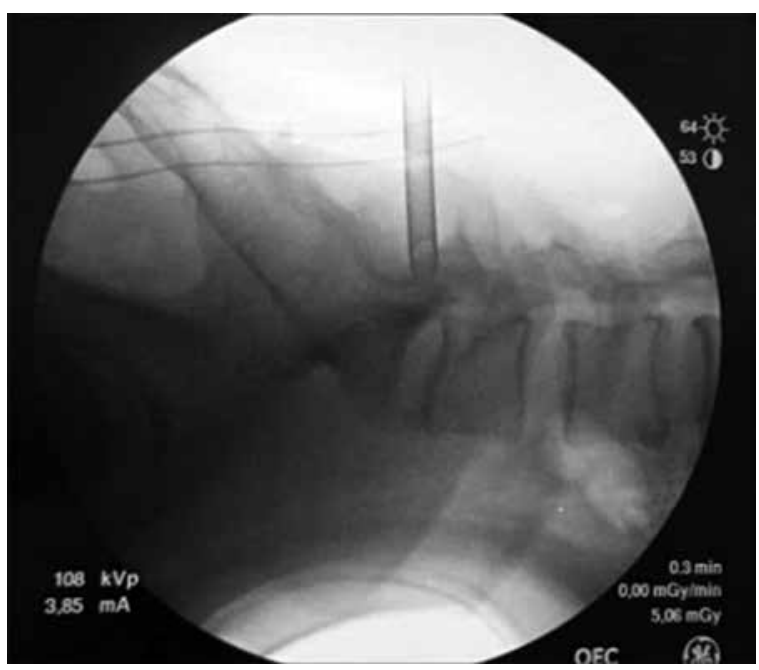

Figura 3 - Confirmação após inserção de canal de trabalho.
Ao final do procedimento, são realizados o inventário da cavidade (Figura 10), a hemostasia e, em nosso serviço, ao término da cirurgia, um curativo de micropore em incisão, permanecendo por 7-15 dias, sem a necessidade de pontos.

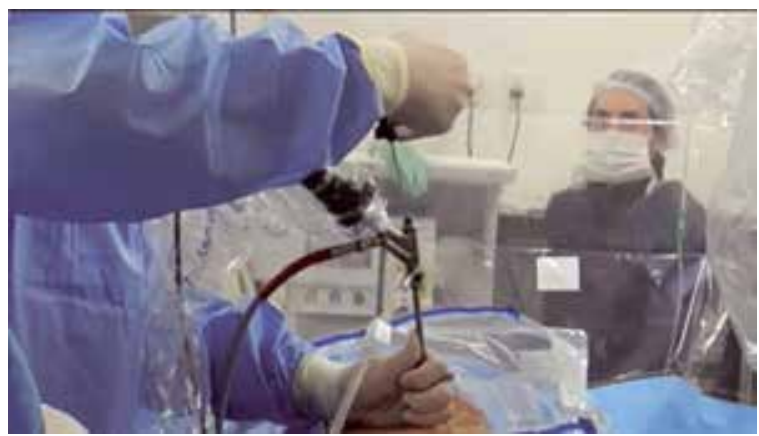

Figura 4 - Óptica no canal de trabalho com início do procedimento cirúrgico.



Figura 5 - Dissecção do ligamento amarelo com bipolar Triguer-Flex.



Figura 6 - Ligamento amarelo aberto. 




Figura 7 - Saca-bocado para ampliar janela.

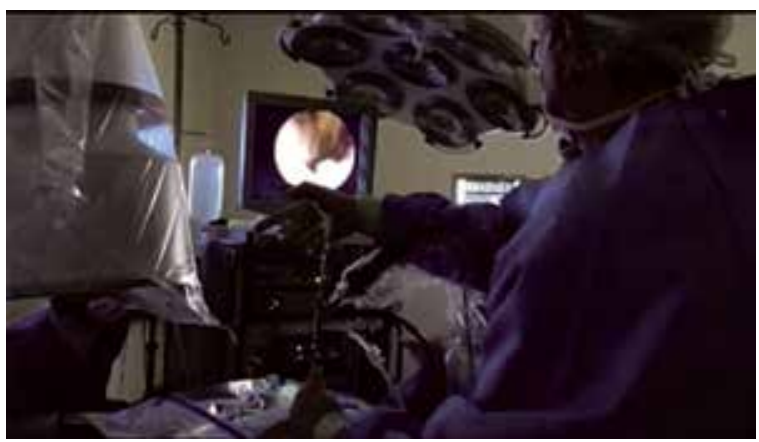

Figura 8 - Movimento de joystick.

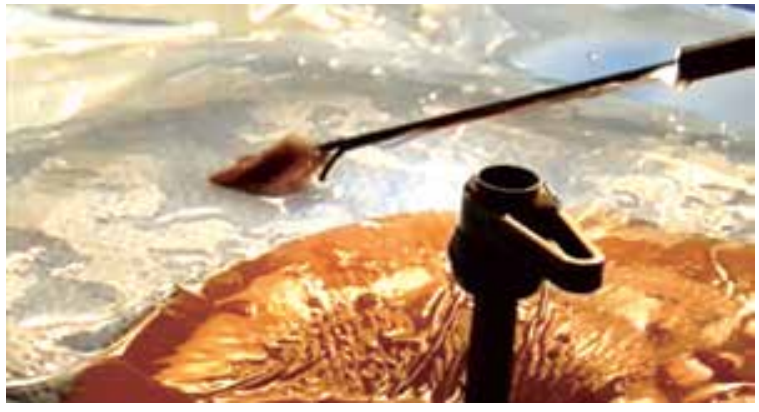

Figura 9 - Retirada da hérnia em conjunto com a óptica.

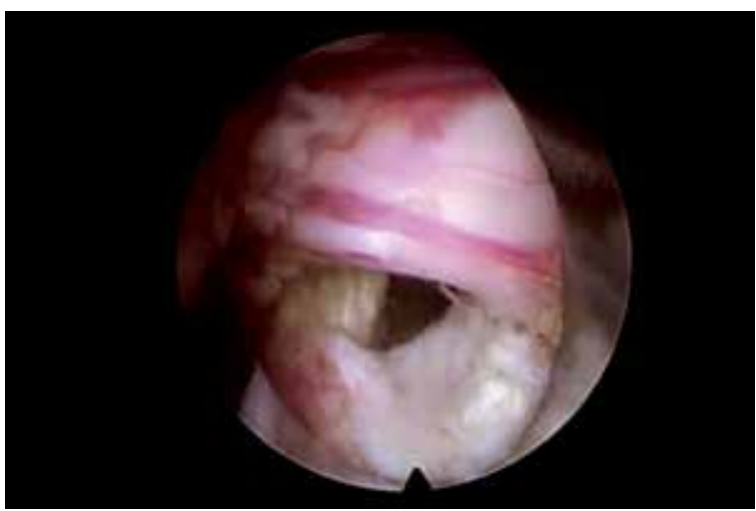

Figura 10 - Ausência de fragmento e visualização da raiz descendente.

\section{Complicações}

Qualquer procedimento cirúrgico está sujeito a complicações, cabendo ao cirurgião tentar minimizar ao máximo a ocorrência delas.

Com a cirurgia vídeo totalmente endoscópica, complicações como sangramento e infecções são minimizadas com o uso de irrigação contínua com soro fisiológico $0,9 \%$, porém as taxas de complicações são as mesmas de uma cirurgia aberta.,

São descritas algumas complicações em diversos artigos científicos. ${ }^{3,5-7}$

A ocorrência de lesões na dura-máter pode ser visualizada rapidamente, porém a tentativa de correção de pequenas fístulas nem sempre é possível, mas pode ser tentada com a cauterização perilesional com retração e consecutiva diminuição do orifício dural, com sucesso significativo na casuística de nosso serviço.

\section{Considerações finais}

Após uma série de mais de 200 casos operados pela técnica vídeo totalmente endoscópica pelo nosso serviço, pudemos avaliar a importância dessa nova técnica como uma arma importante nos dias atuais para tratamento de patologias da coluna vertebral num todo, assim como já utilizado há anos em outras áreas como ortopedia, cirurgia geral, otorrino, cirurgia torácica, urologia, neurocirurgia intracraniana, entre outras.

A curva de aprendizado do cirurgião referente à técnica é um ponto crucial para o sucesso da cirurgia. Inicialmente, ocorre a necessidade de conversão da cirurgia videoendoscópica para a cirurgia aberta, por diversos motivos, como sangramento, tempo excessivo cirúrgico e dificuldade em ressecção das estruturas.

O índice de sucessos e recidivas é semelhante ao da cirurgia aberta, porém com um índice nulo de infecções, em nosso serviço, com a cirurgia vídeo totalmente endoscópica.

O tempo de internação é outro ponto positivo para a nova técnica, pois o paciente é internado em regime de hospital-dia, com alta hospitalar algumas horas após o procedimento cirúrgico.

Com a utilização da videoendoscopia, estamos capacitados a realizar modernos procedimentos de forma eficaz, segura e precisa, sem a necessidade de cirurgia aberta, com o menor índice possível de morbidade e infecção.

\section{Conflito de interesses}

Os autores declaram não haver conflito de interesses. 


\section{Referências}

1. Ruetten S. Full-endoscopic interlaminar lumbar discectomy and spinal decompression. In: Kim DH, Kim KH, Kim YC. Minimally invasive percutaneous spinal techniques. Philadelphia: Elsevier/Saunders; 2010. Disponível em: <http:// www.expertconsultbook.com/expertconsult/ob/book>.

2. Ruetten S, Komp M, Godolias G. A new full-endoscopic technique for the interlaminar operation of lumbar disc herniations using 6-mm endoscopes: prospective 2-year results of 331 patients. Minim Invasive Neurosurg. 2006;49(2):80-7.

3. Hermantin FU, Peters T, Quartararo L, Kambin P. A prospective, randomized study comparing the results of open discectomy with those of video-assisted arthroscopic microdiscectomy. J Bone Joint Surg Am. 1999;81(7):958-65.

4. Andrews DW, Lavyne MH. Retrospective analysis of microsurgical and standard lumbar discectomy. Spine (Phila Pa 1976). 1990;15(4):329-35.
5. Kambin P, Zhou L. History and current status of percutaneous arthroscopic disc surgery. Spine (Phila Pa 1976). 1996;21(Suppl 24):57S-61.

6. Lew SM, Mehalic TF, Fagone KL. Transforaminal percutaneous endoscopic discectomy in the treatment of far-lateral and foraminal lumbar disc herniations. J Neurosurg. 2001;94(Suppl 2):216-20.

7. Weber BR, Grob D, Dvorák J, Müntener M. Posterior surgical approach to the lumbar spine and its effect on the multifidus muscle. Spine (Phila Pa 1976). 1997;22(15):1765-72.

\section{Endereço para correspondência}

Marco Aurélio Moscatelli Alvarenga

Av. Engenheiro Roberto Freire, 9036, ap. 1.003, Ponta Negra

59090-00 - Natal, RN, Brasil

Telefones: (84) 9997-8913/(19) 99618-1305

E-mail: marcomosca13@hotmail.com 